\title{
Impacto de los costos en el margen bruto empresiarial. Caso de la Empresa de mariscos Dispromar
}

\author{
Triviño Mendoza, Brenda Mariela* \\ Garcia Chiriguaya, Elisa Maria* \\ Campos Rocafuerte, Hugo Federico* \\ *Universidad Laica Vicente Rocafuerte de Guayaquil Facultad de \\ Administración Contabilidad y Auditoria \\ E-mail: elisa-garcia@hotmail.com
}

Recibido: 5 diciembre de 2019 Aprobado: 22 diciembre de 2019

\begin{abstract}
Resumen
El presente artículo tiene como objetivo la determinación de los costos y su incidencia en el margen bruto, efectuada desde un enfoque aplicativo a la información relacionada con los costos de procesamiento y el beneficio directo de la actividad de la empresa. Con su nombre comercial la empresa Dispromar fue el objeto de este estudio, se identificó la necesidad de determinar adecuadamente el costo real de procesamiento del producto, habiéndose detectado que el limitado conocimiento en la asignación de costos afecta directamente al margen bruto. Al no contar con un manual de políticas y procedimientos que establezca las actividades, la información no es llevada de forma idónea, provocando problemas desde la adquisición y utilización de materia prima directa, mano de obra directa y costos indirectos de producción y venta. La rotación del producto es inmediata al ser perecible, no existiendo además un adecuado registro operativo de su inventario, provocando inconsistencias en sus registros. De la metodología de este trabajo de investigación descriptivo es de carácter mixto; es decir, abarca un enfoque cualitativo y cuantitativo ya que con su debida interpretación se permitirá mostrar el cumplimiento de los objetivos planteados. Los resultados obtenidos demuestran una influencia positiva en el precio final del producto, además de tener claridad acerca de nuevas inversiones a realizarse. Se espera que pueda servir de guía para futuros investigadores que puedan aplicar normativas internacionales eliminando la improvisación
\end{abstract}

Palabras claves: Costos, Estrategias de mercado, Ventas, Contabilidad, Producción 


\title{
Impact of costs on gross empresiarial margin. Case of the Dispromar Seafood Company
}

\begin{abstract}
The purpose of this article is to determine the costs and their impact on the gross margin, made from an application approach to information related to the processing costs and the direct benefit of the company's activity. With its commercial name the company Dispromar was the object of this study, the need to properly determine the real cost of processing the product was identified, having found that the limited knowledge in the allocation of costs directly affects the gross margin. By not having a manual of policies and procedures that establish the activities, the information is not carried out in an appropriate way, causing problems from the acquisition and use of direct raw material, direct labor and indirect costs of production and sale. The rotation of the product is immediate because it is perishable, and there is no adequate operational record of its inventory, causing inconsistencies in its records. The methodology of this descriptive research work is mixed; that is, it encompasses a qualitative and quantitative approach since with its proper interpretation it will be possible to show the fulfillment of the objectives set. The results obtained demonstrate a positive influence on the final price of the product, in addition to being clear about future investments to be made. It is hoped that it can serve as a guide for future researchers who can apply international regulations eliminating improvisation
\end{abstract}

Keywords: Costs, Market strategies, Sales, Accounting, Production

\section{Introducción}

El análisis de costos que se realiza en esta investigación está relacionada con la necesidad de determinar adecuadamente el costo real de procesamiento del producto, habiéndose detectado que el limitado conocimiento en la asignación de costos afecta directamente al margen bruto. Al no contar con un manual de políticas y procedimientos que establezca las actividades, la información no es llevada de forma idónea, provocando problemas desde la adquisición y utilización de materia prima directa.

El determinar los costos y su incidencia en el margen bruto de la empresa, se agrega un valor a la información relacionada con los costos de procesamiento políticas y procedimientos de las actividades que realiza DISPROMAR y el beneficio es directo a la actividad de la entidad.

La contabilidad de costos es de suma importancia para las empresas, especialmente para aquellas que realizan procesos de transformación de materia prima, al brindar una visión amplia y clara de lo que invierte en la elaboración de un producto o servicio para así conocer la utilidad o pérdida que genera su comercialización. (Arredondo, 2015).

Al tener la información real de la empresa se puede interpretar la misma y así tomar decisiones acertadas que permitan implementar estrategias para optimizar los costos de producción, para mejorar la administración, tomar decisiones inteligentes y optimizar los gastos en las diferentes áreas que operan en la empresa.

La precisa determinación del precio de un producto permitirá conocer la rentabilidad real de los mismos, correlacionados con los precios que se manejan en el mercado; esto contribuirá no solo en darnos cuenta de la rentabilidad general de la inversión realizada, sino también

Triviño Mendoza, Brenda Mariela; Garcia Chiriguaya, Elisa Maria \& Campos Rocafuerte, Hugo Federico 
del rendimiento verdadero en dicha actividad a la que se dedica la empresa.

Según (Remache, 2015). Este sistema consiste en dividir en el paso en procesos por los cuales pasan los materiales hasta convertirse en artículos terminados. Sistema también conocido de costos por departamentos en las cuales pasan los materiales y los costos durante un período contable.

Este sistema de costos permite controlar la producción de unidades iguales que se realizan en grandes cantidades y de manera continua hasta que se obtiene el producto final. (Deakin, 2046).

De acuerdo al control de costos, "este sistema de costos por procesos trabaja con costos reales e históricos, por lo cual los costos unitarios se calculan al final del período contable y para ese tiempo ya se conocen los costos generales de fabricación realmente incurridos" (Remache, 2015).

Así mismo, se establece los costos de cada uno de los procesos productivos y en caso de ser comunes tienen que ser distribuidos.

\section{Objetivos}

Los objetivos de esta investigación son:

Determinar un sistema de costos de producción para la empresa de mariscos Dispromar que permita medir la incidencia en el margen bruto para la toma de decisiones.

De los objetivos específicos que se plantearon son:

- Establecer parámetros para el registro y control de los costos de procesamiento que permitan medir la situación financiera de la empresa de Mariscos Dispromar

- Introducir procesos para el cálculo de los costos de producción que permitan mejorar el margen bruto de rentabilidad de la empresa de Mariscos Dispromar

- Definir un sistema de costo que debe seguir la empresa de Mariscos Dispromar que le permita tomar decisiones.

\section{Desarrollo}

La importancia de la contabilidad de costos no radica en la información obtenida, sino en la interpretación que se hace de ella y la forma en que se utiliza para aumentar las ganancias mediante la optimización de los procesos de producción. Es decir, tener la información de la situación real de la empresa, puede ser interpretada $\mathrm{y}$, por lo tanto, tomar decisiones acertadas que permitan implementar diferentes estrategias para optimizar los costos de producción, como la reducción de personal, la adquisición de maquinaria más eficiente, la implementación de materiales más baratos, entre otros (Pérez, 2015).

La importancia de que las empresas comerciales y los proveedores de servicios lleven este tipo de contabilidad que no implica más trabajo y que representa información valiosa para mejorar la gestión, tomar decisiones inteligentes y optimizar los gastos en las diferentes áreas que operan en la empresa.

La información contable es acumulativa por departamentos u otros centros de costo y se 
promedia entre toda la producción que se realizó en el centro de costo específico. En un sistema de órdenes de trabajo específicas, los materiales, la mano de obra y los gastos generales de fabricación se acumulan por órdenes o lotes. La contabilidad de costos de proceso es utilizada por compañías que fabrican productos a través de un flujo más o menos continuo, sin referencia a pedidos o lotes específicos (González, 2016).

\section{Objetivos de la determinación de costos}

En el contexto de la investigación del sistema especializado de contabilidad se considera como objetivos principales:

- Determinar el costo de los inventarios de productos.

- Definir el costo de los productos vendidos

- Calcular la utilidad o pérdida y poder reparar el estado de rentas y gastos.

- Generar una herramienta útil para la planeación y control de los costes de producción

- Constituir una fuente de información de costos para estudios económicos y toma de decisiones relacionas con producción e inversiones.

Los costos de inventario son costos relacionados con el almacenamiento y mantenimiento del inventario por un cierto período de tiempo. En general, los costos de inventario se describen como un porcentaje del valor del inventario (Castillo, 2016).

Por lo tanto, la evaluación de los costos de inventario es esencial y tiene un impacto en las finanzas de la empresa, así como en su gestión. Ayuda a las empresas a determinar cuánta ganancia pueden obtener del inventario, cómo pueden reducir los costos, dónde se pueden hacer cambios, qué proveedores o qué artículos deben elegirse, cómo se debe asignar el capital, etc.

El costo de los productos vendidos es una línea de crédito en el estado de resultados de una empresa. Los gastos en materias primas y costos de producción para crear un producto terminado (Horngren, 2015)

\section{Elementos del costo}

Los elementos que se consideran necesarios de forma general, para establecer el costo son:

- Materia prima

- Mano de obra

- Costos indirectos de fabricación

La materia prima comprende todos los elementos que se utilizan en la elaboración de un producto final. La materia prima cuando se puede identificar con los productos terminados se llama materia prima directa, mientras que cuando no se puede identificar con los productos terminados se considera materia prima indirecta (Jimenez, 2015).

La mano de obra se entiende como todos los salarios, beneficios sociales, contribuciones parafiscales y otros conceptos laborales, que se pagan a las personas que participan directa o indirectamente en la producción del bien o la

Triviño Mendoza, Brenda Mariela; Garcia Chiriguaya, Elisa Maria \& Campos Rocafuerte, Hugo Federico 
prestación del servicio (Cárdenas, 2016)

Cada empresa para cumplir sus objetivos requiere un esfuerzo humano, ya sea físico o mental, para transformar la materia prima en productos terminados. Por lo tanto, se afirma que sin trabajo no hay producción, ya que todo tiene un proceso de creación en el que el trabajo está involucrado, ya sea directa o indirectamente.

Los costos indirectos de fabricación son aquellos costos que una fábrica debe cubrir para la fabricación de un producto, además de los materiales y la mano de obra directa (Arias, 2015).

Estos son costos que están relacionados con toda la operación de la empresa y exceden el proceso de fabricación de un producto específico. Reconocer estos costos es esencial, ya que son necesarios para poder identificar el costo real de fabricación de un producto y de esta manera establecer el precio para el consumidor y los beneficios esperados de la comercialización.

\section{Metodología}

Este artículo está basado en una investigación de tipo descriptiva, en el que se pudo detallar las distintas variables que abarcaron el tema. La investigación descriptiva fue necesaria para conocer las características, situaciones, eventos, políticas, normas y demás mecanismos que la empresa aplicó, es decir al describir y desglosar las actividades del área nos permitió demostrar los resultados e impactos favorables y desfavorables para la empresa.

El enfoque de la investigación descriptiva fue de carácter mixto, abarcó un enfoque cualitativo y cuantitativo, lo cual permitió mostrar el cumplimiento de los objetivos planteados al inicio de la investigación.

Como parte de las técnicas que se utilizaron también fue la entrevista, mediante una conversación entre los autores y personas que se establecieron en la muestra, posteriormente fue sistematizada para evidenciar y sustentar la información proporcionada.

\section{Resultados y discusión}

Dispromar es una empresa que no cuenta con procesos contables definidos, la contabilidad que realiza es proporcionada por un ente externo cada vez que lo requiere la empresa con el fin de cubrir las obligaciones con entidades de control gubernamental.

El objetivo es determinar los costos de producción y venta que debe seguir la empresa de Mariscos DISPROMAR S.A. desde la adquisición y utilización de materia prima directa, mano de obra directa y costos indirectos de producción y venta.

\section{Determinación de la producción y venta}

Para determinar la venta se toma como referencia la cantidad producida mensualmente durante el año 2017. En la siguiente tabla se muestra el detalle de producción anual de la empresa (Dispromar, 2017) 
Tabla 1

Venta mensual de libras de camarones. 2017

\begin{tabular}{|l|c|lr|}
\hline \multicolumn{3}{|c|}{ Resumen por camarones } \\
\hline \multicolumn{1}{|c|}{ Mes } & Total de libras & \multicolumn{2}{c|}{$\begin{array}{c}\text { Precio por } \\
\text { libra }\end{array}$} \\
\hline Enero & 6000 & $\$$ & 3,86 \\
\hline Febrero & 5500 & $\$$ & 3,95 \\
\hline Marzo & 6600 & $\$$ & 3,93 \\
\hline Abril & 4000 & $\$$ & 3,74 \\
\hline Mayo & 4700 & $\$$ & 3,85 \\
\hline Junio & 4800 & $\$$ & 3,84 \\
\hline Julio & 5300 & $\$$ & 3,90 \\
\hline Agosto & 4500 & $\$$ & 3,80 \\
\hline Septiembre & 5850 & $\$$ & 3,75 \\
\hline Octubre & 5700 & $\$$ & 3,90 \\
\hline Noviembre & 5000 & $\$$ & 3,81 \\
\hline Diciembre & 6250 & $\$$ & 3,98 \\
\hline
\end{tabular}

Fuente: DISPROMAR (2018)

De acuerdo a los datos presentados se observa que el promedio de venta mensual por libras es 6.000 libras, con un precio aproximado de 3.90 por libra danto una rentabilidad promedio de $\$ 23,400.00$

\section{Materia Prima}

La materia prima se clasifica en: material directo y material indirecto. La materia prima de este proceso son los camarones blancos, talla $31-35$, es el material directo y el hielo, carnal químico y sal en grano son el material indirecto. La adquisición de esta materia se describe en el siguiente proceso al igual que el material.

En el siguiente gráfico se describe la forma para adquirir o comprar la materia prima y los registros contables de cada uno

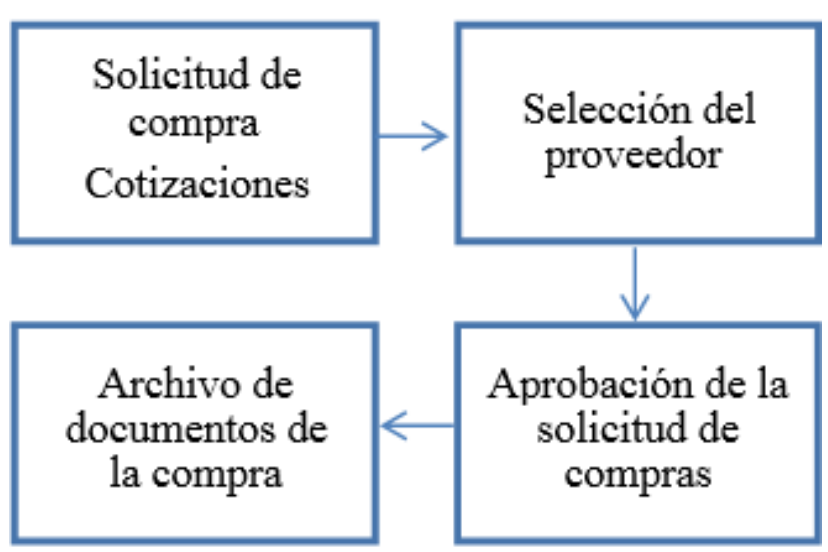

Figura 1: Flujograma del proceso de adquisición y utilización de la materia prima

\section{Mano de obra directa}

El proceso contable de la mano de obra comprende lo siguiente:

Triviño Mendoza, Brenda Mariela; Garcia Chiriguaya, Elisa Maria \& 
Tabla 2

Proceso mano de obra

\begin{tabular}{|r|c|r|r|r|r|r|}
\hline \multicolumn{7}{|c|}{ MANO DE OBRA. DISPROMAR } \\
\hline $\mathbf{N}^{\mathbf{0}}$ & NOMBRE & $\begin{array}{c}\text { LBS. } \\
\text { PELADAS }\end{array}$ & V. LIBRAS & H. EMPAQUE & V. HORA & TOTAL \\
\hline 1 & OPERADOR & 500 & $\$ 0,25$ & 1 & $\$ 2,50$ & $\$ 127,50$ \\
\hline 2 & OPERADOR & 450 & $\$ 0,25$ & 1 & $\$ 2,50$ & $\$ 115,00$ \\
\hline 3 & OPERADOR & 600 & $\$ 0,25$ & 1 & $\$ 2,50$ & $\$ 152,50$ \\
\hline 4 & OPERADOR & 450 & $\$ 0,25$ & 1 & $\$ 2,50$ & $\$ 115,00$ \\
\hline 5 & OPERADOR & 500 & $\$ 0,25$ & 1 & $\$ 2,50$ & $\$ 127,50$ \\
\hline 6 & OPERADOR & 400 & $\$ 0,25$ & 1 & $\$ 2,50$ & $\$ 102,50$ \\
\hline 7 & OPERADOR & 600 & $\$ 0,25$ & 1 & $\$ 2,50$ & $\$ 152,50$ \\
\hline 8 & OPERADOR & 400 & $\$ 0,25$ & 1 & $\$ 2,50$ & $\$ 102,50$ \\
\hline 9 & OPERADOR & 450 & $\$ 0,25$ & 1 & $\$ 2,50$ & $\$ 115,00$ \\
\hline 10 & OPERADOR & 550 & $\$ 0,25$ & 1 & $\$ 2,50$ & $\$ 140,00$ \\
\hline 11 & OPERADOR & 500 & $\$ 0,25$ & 1 & $\$ 2,50$ & $\$ 127,50$ \\
\hline 12 & OPERADOR & 600 & $\$ 0,25$ & 1 & $\$ 2,50$ & $\$ 152,50$ \\
\hline & TOTAL DE PAGO & & & & & $\$ 1.530,00$ \\
\hline
\end{tabular}

Fuente: DISPROMAR (2018)

La fuerza laboral de la empresa de Mariscos DISPROMAR se compone principalmente por los trabajadores encargados del empacado de camarón. Por lo que es relevante considerar los factores vinculados al seguimiento, control, clasificación y contabilización de la mano de obra directa.

\section{Costos indirectos de fabricación. (CIF)}

Son aquellos costos que debe cubrir la empresa para la producción de camarones, aparte de la materia prima y la mano de obra y se relacionan con todo el funcionamiento de la empresa Dispromar y estos son:

REVISTA DE INVESTIGACIÓN SIGMA / Vol. 06, Nº 2, 2019 (pág. 25-35) 
Tabla 3

Costos indirectos de fabricación valores totales

\begin{tabular}{|c|c|c|c|}
\hline \multicolumn{4}{|c|}{ Costos Indirectos de fabricación } \\
\hline Concepto & Cantidad & Costo/u & TOTAL \\
\hline \multicolumn{4}{|l|}{ SUMINISTROS } \\
\hline Empaque (unidades) & 6,000 & 0.036 & 220.25 \\
\hline \multicolumn{4}{|c|}{ SERVICIOS PROFESIONALES } \\
\hline Supervisor (horas) & 10 & 11.82 & 118.20 \\
\hline \multicolumn{4}{|l|}{ MANTENIMIENTO } \\
\hline Mantenimiento y reparación & & 150.00 & 150.00 \\
\hline Visita técnica (horas) & 2 & 12.50 & 25.00 \\
\hline Mallas & & 36.33 & 36.33 \\
\hline Equipos de protección & 12 & 4.75 & 57.00 \\
\hline Depreciación PPE & & 25.42 & 25.42 \\
\hline \multicolumn{3}{|r|}{ Total } & 632.20 \\
\hline
\end{tabular}

Fuente: DISPROMAR (2018)

En este cuadro se puede observar el CIF, tomando como referencia los datos obtenidos como ejercicio de la empresa DISPROMAR en al año 2017, se muestra que el CIF se centra en suministros, servicios profesionales y mantenimiento, durante el año el CIF mantiene una variación no mayor que se toma como referencia un costo aproximado de 650.00

\section{Estados de costos de producción}

Concluido el proceso contable del personal de la empresa camaronera DISPROMAR, se procede a elaborar el estado de costos de producción, ya que se resume toda la información, registrando los tres elementos del costo: materia prima, mano de obra y costos indirectos de fabricación.

Triviño Mendoza, Brenda Mariela; Garcia Chiriguaya, Elisa Maria \& 
Tabla 4

Análisis hoja de costos mensual

\begin{tabular}{|c|c|c|c|c|c|c|c|c|}
\hline MES & $\begin{array}{c}\text { Materia } \\
\text { prima }\end{array}$ & $\begin{array}{c}\text { Mano } \\
\text { de obra } \\
\text { directa }\end{array}$ & CIF & $\begin{array}{c}\text { Total de } \\
\text { costos de } \\
\text { producción }\end{array}$ & $\begin{array}{c}\text { Margen de } \\
\text { rentabilidad } \\
\mathbf{2 0 \%}\end{array}$ & $\begin{array}{c}\text { Total } \\
\text { producido } \\
\text { con } \\
\text { ganancia }\end{array}$ & $\begin{array}{c}\text { Total de } \\
\text { libras } \\
\text { producidas }\end{array}$ & $\begin{array}{c}\text { Valor } \\
\text { por } \\
\text { libra }\end{array}$ \\
\hline 1 & $18,726.20$ & $1,537.50$ & 632.25 & $20,895.95$ & $4,179.19$ & $25,075.14$ & 6.500 & 3.86 \\
\hline 2 & $17,153.00$ & $1,407.50$ & 547.25 & 19.107 .75 & $3,821.55$ & $22,929.30$ & 5.800 & 3.95 \\
\hline 3 & $20,589.27$ & $1,692.50$ & 648.75 & 22.930 .52 & $4,586.10$ & $27,516.62$ & 7.000 & 3.93 \\
\hline 4 & $12,499.74$ & $1,030.00$ & 507.25 & $14,036.99$ & $2,807.40$ & $16,844.39$ & 4,500 & 3.74 \\
\hline 5 & $14,678.31$ & $1,207.50$ & 467.55 & $16,353.36$ & $3,270.67$ & $19,624.03$ & 5.100 & 3.85 \\
\hline 6 & 14.977 .50 & $1,232.50$ & 607.25 & $16,817.25$ & $3,363.45$ & $20,180.70$ & 5.250 & 3.84 \\
\hline 7 & $16,547.16$ & $1,360.00$ & 632.25 & $18,539.41$ & $3,707.88$ & $22,247.29$ & 5.700 & 3.90 \\
\hline 8 & $14,056.35$ & $1,160.00$ & 607.25 & $15,823.60$ & $3,164.72$ & $18,988.32$ & 5.000 & 3,80 \\
\hline 9 & $18,253.93$ & $1,497.50$ & 552.25 & 20.303 .68 & $4,060.74$ & $24,364.42$ & 6.500 & 3.75 \\
\hline 10 & $17,790.23$ & $1,460.00$ & 552.25 & $19,802.48$ & $3,960.50$ & $23,762.98$ & 6,100 & 3,90 \\
\hline 11 & 15.618 .93 & $1,285.00$ & 562.25 & $17,466.18$ & $3,493.24$ & 20.959 .42 & 5.500 & 3.81 \\
\hline 12 & $19,510.24$ & $1,605.00$ & 467.25 & 21.582 .49 & $4,316.50$ & $25,898.99$ & 6.500 & 3.98 \\
\hline & & & & & & & & \\
\hline
\end{tabular}

Fuente: Elaboración propia

\section{Análisis de las variaciones}

A partir del análisis de ambos sistema de costos se presenta las variaciones existentes en cada proceso, como se muestra a continuación.

\section{Tabla 5}

Análisis de las variaciones

\begin{tabular}{|c|c|r|r|}
\hline $\begin{array}{c}\text { SISTEMA DE COSTO } \\
\text { DE LA ENTIDAD }\end{array}$ & $\begin{array}{c}\text { SISTEMA DE COSTOS } \\
\text { PROPUESTO }\end{array}$ & VARIACIÓN & PORCENTAJE \\
\hline \multicolumn{3}{|c|}{ MATERIA PRIMA } & $16.6 \%$ \\
\hline$\$ 18.726,20$ & $\$ 15.606,30$ & $\$ 3.119,90$ & \\
\hline \multicolumn{3}{|c|}{ MANO DE OBRA DIRECTA } & \\
\hline$\$ 1.537,50$ & $\$ 1.530,00$ & $\$ 7,50$ & $0.48 \%$ \\
\hline
\end{tabular}

Fuente: Elaboración propia

De acuerdo a los resultados, el área para la elaboración del libro mayor. Si se realiza financiera no lleva adecuado control contable, a un informe contable que es facilitado por la pesar de que llevan el libro diario no lo utilizan contadora, sin embargo, este informe no se utiliza

REVISTA DE INVESTIGACIÓN SIGMA / Vol. 06, Nº 2, 2019 (pág. 25-35) 
para la clasificación y análisis de los ingresos, egresos y gastos operativos de la empresa, lo que no permite la elaboración del presupuesto.

Si bien es cierto han llevado el libro diario no de forma adecuada, pero se lo ha llevado. No se ha dado paso a la elaboración del libro mayor, lo cual no permite el balance de cada cuenta, ajustes, cierres y balance general que conlleva a tener un control anual dela empresa, su estado situacional, el margen bruto de rentabilidad para poder realizar una adecuada proyección de gastos e ingresos.

En el estado de resultados proyectado, aplicado en el análisis de la producción y venta en referencia a la materia prima se produjo una variación del $16.6 \%$ esto representa un incremento en el nivel de producción de la empresa. En referencia a la mano de obra directa no existe mayor variación por las características de pago de mano de obra y esta es por hora y por libra.

De esta manera se puede observar, como el control del proceso contable ayuda a optimizar los costos del proceso productivo en la compra y utilización de la materia prima.

\section{Conclusiones}

La Empresa Dispromar, no aplica un sistema de costos por procesos que le permita tener una información contable de calidad dificultando la obtención de resultados reales sobre la posición financiera de la empresa y sus operaciones realizadas impidiendo la toma de decisiones adecuadas y oportunas para la administración.
En el estado de resultados proyectado, aplicado en el análisis de la producción y venta en referencia a la materia prima se produjo una variación del $16.6 \%$ esto representa un incremento en el nivel de producción de la empresa. En referencia a la mano de obra directa no existe mayor variación por las características de pago de mano de obra y esta es por hora y por libra.

De esta manera se puede observar, como el control del proceso contable ayuda a optimizar los costos del proceso productivo en la compra y utilización de la materia prima.

\section{Recomendaciones}

Con la información mostrada en las conclusiones se pueden realizar varias recomendaciones que permitirán mejorar la situación de la empresa:

Realizar la implementación según el formato establecido en el diseño del sistema contable de costos por procesos, ya que le permitirá al propietario conocer los resultados de cada período contable y conocer el beneficio que está obteniendo en la actividad que se desarrolla

Establecer un control sobre los inventarios de entrada y salidas de los distintos productos este se debe realizar de forma automatizada para evitar riesgos y ayudar el crecimiento económico de la empresa.

Implementar un registro de control para los trabajadores, de preferencia automatizado de forma de biométrico, que permitirá tener control en el gasto administrativo de roles de pago. 
Realizar una inversión tecnológica en la empresa en lo concerniente a automatizaciones de las operaciones de la empresa, esto permitirá el fortalecimiento y ganar mayor porcentaje de poder de mercado, y así el incremento de más productos para la venta

\section{Referencias bibliográficas}

Arias, L. (2015). la distribución de costos indirectos de fabricación, factor clave al costear productos. Colombia.

Arredondo, M. (2015). Contabilidad y análisis de costos. México D.F.: Patria. Cárdenas, R. (2016). Costos. Instituto mexicano de contadores públicos. México.

Deakin, E. (2016). Principios de práctica de contabilidad. México D.F.: MC Graw Hill.

DISPROMAR. (2017). promedio mensual de ventas de camarón. Guayaquil. González, D. (2016). Contabilidad de costos. México D.F.: Ecafsa.

Horngren, C. (2015). Contabilidad de costos. Un enfoque gerencial ( decimo cuarta edición). méxico: Pearson.

Jimenez, W. (2015). Contabilidad de costos. Fundación para la educación superior de San mateo. Colombia: FESM.

Lino, A., \& Jalón, A. (2015). Auditoría FInanciera a los Costos y Gastos de la Camaronera Imperial "CAMARIMPE" S.A. de la CIudad de Guayaquil durante el año 2013. Guayaquil: Universidad Politécnica Salesiana .
Pérez, L. (2015). Contabilidad de costos. México D.F.: MC Graw Hill.

PROECUADOR. (2015). Acuacultura. Obtenido de http://www. proecuador.gob.ec/ compradores/ofertaexportable/fishingand- aquaculture/

Remache, D. (2015). Implementación de sistemas de costos por procesos de producción de la empresa shinatex s.a.ubicada en la ciudad de Quito. Obtenido de Universidad Central del Ecuador: http://www.dspace. uce.edu.ec/bitstream/25000/9093/1/TUCE- 0003-CA202-2015.pdf 